Notwithstanding these important unresolved questions, the Zhu et al. (5) study should open up a new area of investigation as additional studies probe the connections between $\beta_{1}$-adrenergic receptor-mediated signaling and CaMKII-dependent effects. If future studies support the proposal by Zhu et al., that one of the consequences of prolonged $\beta_{1}$-adrenergic receptor stimulation is cardiomyocyte cell death via a CaMKII-mediated pathway, it will indeed be an important contribution to our understanding of the pathogenesis of heart failure that will suggest novel therapeutic targets.

1. Molkentin, J.D., et al. 1998. A calcineurindependent transcriptional pathway for cardiac hypertrophy. Cell. 93:215-228.

2. Marks, A.R., Reiken, S., and Marx, S.O. 2002. Progression of heart failure: is protein kinase a hyperphosphorylation of the ryanodine receptor a contributing factor? Circulation. 105:272-275.

3. Chidsey, C.A., Harrison, D.C., and Braunwald, E. 1962. Augmentation of plasma norepinephrine response to exercise in patients with congestive heart failure. N. Engl. J. Med. 267:650.

4. Marx, S.O., et al. 2000. PKA phosphorylation dissociates FKBP12.6 from the calcium release channel (ryanodine receptor): defective regulation in failing hearts. Cell. 101:365-376.

5. Zhu, W.-Z., et al. 2003. Linkage of $\beta_{1}$-adrenergic stimulation to apoptotic heart cell death through protein kinase $\mathrm{A}$-independent activation of $\mathrm{Ca}^{2+} /$ calmodulin kinase II. J. Clin. Invest. 111:617-625. doi:10.1172/JCI200316326.

6. Luttrell, L.M., et al. 1999. Beta-arrestin-dependent formation of beta2 adrenergic receptor-Src protein kinase complexes. Science. 283:655-661.

7. Zaccolo, M., Magalhaes, P., and Pozzan, T. 2002 Compartmentalisation of cAMP and $\mathrm{Ca}(2+)$ signals. Curr. Opin. Cell. Biol. 14:160-166.

\title{
Soluble VEGF receptor Flt1: the elusive preeclampsia factor discovered?
}

\section{Aernout Luttun and Peter Carmeliet}

The Center for Transgene Technology and Gene Therapy, Flanders Interuniversitary Institute for Biotechnology, Katholieke Universiteit Leuven, Leuven, Belgium

J. Clin. Invest. 111:600-602 (2003). doi:10.1172/JCI200318015.

The occurrence of seizures (eclampsia, from the Greek "eklampsis," sudden flashing) has been a long-known and feared complication of pregnancy, often killing both mother and child. Preeclampsia, or the condition preceding full-blown eclampsia, affects up to $5 \%$ of pregnant women and is diagnosed by the onset of hypertension and proteinuria in the second trimester (1). Preeclampsia may eventually progress to glomerular malfunction, thrombocytopenia, liver and brain edema, and associated lifethreatening seizures (2) (Figure 1). Preeclampsia has been sometimes

\footnotetext{
Address correspondence to: Peter Carmeliet, Center for Transgene Technology and Gene Therapy, Flanders Interuniversitary Institute for Biotechnology, Katholieke Universiteit Leuven, Campus Gasthuisberg, Herestraat 49, B-3000, Leuven, Belgium.

Phone: 32-16-34-57-72; Fax: 32-16-34-59-90; E-mail: peter.carmeliet@med.kuleuven.ac.be. Conflict of interest: The authors have declared that no conflict of interest exists. Nonstandard abbreviations used: placental growth factor (PlGF); fms-like tyrosine kinase 1 (Flt1); soluble Flt1 (sFlt1).
}

termed the "disease of theories," as several models for its pathogenesis have been proposed. But, as of today, no satisfactory unifying hypothesis has emerged (1). The restricted occurrence of preeclampsia to humans and primates and the lack of a suitable animal model have hampered the understanding of its pathogenesis (3). In this issue of the JCI, S.E. Maynard et al. (4) report the novel insight that circulating levels of two angiogenic growth factors, VEGF and placental growth factor (PlGF), may play a more important role than previously believed. In particular, the authors propose that, in pregnant women with preeclampsia, the placenta produces elevated levels of the soluble fms-like tyrosine kinase 1 (sFlt 1 ) receptor, which captures free VEGF and PIGF. As a result, the normal vasculature in the kidney, brain, lungs, and other organs is deprived of essential survival and maintenance signals and becomes dysfunctional (Figure 1). As the authors show in their rodent model, this may lead to the development of hypertension and renal dis- ease, reminiscent of preeclampsia in humans. In another study in this issue, V. Eremina et al. (5) provide additional evidence for a critical role of VEGF in renal disease during preeclampsia. These authors demonstrate that mice lacking one VEGF allele in renal podocytes develop the typical renal pathology found in pregnant women with preeclampsia. These studies therefore shed unprecedented light on the pathogenesis of preeclampsia and offer novel therapeutic opportunities for this disease.

\section{sFlt1: a likely candidate preeclampsia factor}

For the fetus to develop normally, it must receive sufficient oxygen and nutrients (6). These are supplied via the maternal spiral arteries in the uterus. During normal pregnancy, cytotrophoblasts convert from an epithelial to an endothelial phenotype (a process termed pseudo-vasculogenesis) and invade maternal spiral arteries. This vascular remodeling increases the bulk flow and the supply of nutrients and oxygen to the fetus by the end of the first trimester $(7,8)$ (Figure 1). Vascular factors such as VEGF, angiopoietins, and ephrins have been implicated in this process (7). In preeclampsia, pseudo-vasculogenesis is defective, and the resultant placental ischemia has been proposed to trigger the release of unknown placenta-derived factors. The latter would induce systemic endothelial dysfunction and thereby contribute to the renal, cardiovascular, and neurological defects of preeclampsia (Figure 1). Despite intensive efforts, the precise nature of the placenta-derived 

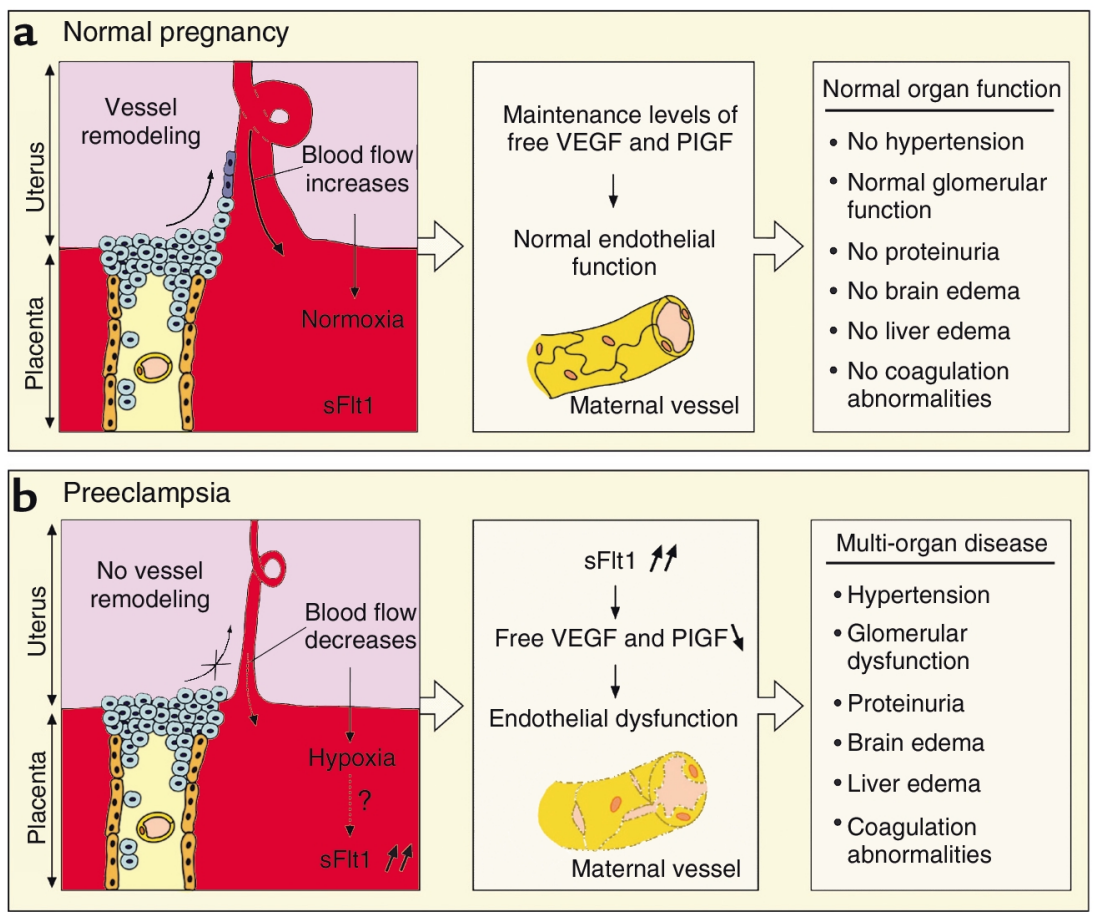

Figure 1

Hypothesis on the role of sFlt1 in preeclampsia. (a) During normal pregnancy, the uterine spiral arteries are infiltrated and remodeled by endovascular invasive trophoblasts, thereby increasing blood flow significantly in order to meet the oxygen and nutrient demands of the fetus. (b) In the placenta of preeclamptic women, trophoblast invasion does not occur and blood flow is reduced, resulting in placental hypoxia. In addition, increased amounts of soluble Flt1 (sFlt1) are produced by the placenta and scavenge VEGF and PIGF, thereby lowering circulating levels of unbound VEGF and PIGF. This altered balance causes generalized endothelial dysfunction, resulting in multi-organ disease. It remains unknown whether hypoxia is the trigger for stimulating sFlt1 secretion in the placenta of preeclamptic mothers and whether the higher sFlt 1 levels interfere with trophoblast invasion and spiral artery remodeling.

preeclampsia factors has remained enigmatic for years.

In their study, Maynard et al. (4) may have discovered a likely candidate preeclampsia factor. Indeed, having used gene profiling, these authors report that the placenta of pregnant women with preeclampsia produced increased levels of sFlt1. Flt1, binding VEGF and its homologue PIGF, exists in two forms: a membrane-bound receptor tyrosine kinase which transmits angiogenic signals (Flt 1 ), and a soluble secreted ectodomain which only captures VEGF and PlGF (sFlt1). As sFlt1 lacks a cytosolic domain, its function is restricted to regulating (reducing) the levels of free VEGF and PlGF available to signal via intact Flt1 and fetal liver kinase-1. Even though sFlt1 may be one of the few, if not the only, inhibitor of VEGF known today, it remained unknown whether it might contribute to any sort of human disease. prodocytes drop 50\%, glomerular endothelial cells swell, capillary loops collapse, and proteinuria develops as in preeclampsia. We previously demonstrated that absence of the VEGF'164 and VEGF'188 isoforms impairs glomerular filtration (9). Endothelial dysfunction may also deregulate hemostasis and trigger thrombocytopenia. By inducing vasodilation, VEGF also induces hypotension, and thus lower circulating VEGF levels will cause elevated blood pressure, another hallmark of preeclampsia. Thus, the sFlt 1 hypothesis allows the proposal of a unifying model, explaining, perhaps not all, but at least several, of the hallmark symptoms of preeclampsia.

As insightful as these studies are, they do not address the question as to what upregulates sFlt 1 expression in the placenta in preeclampsia. Hypoxia upregulates Flt1 expression and, since the placenta is hypoxic in pregnant women with preeclampsia, could be responsible (Figure 1). It also remains to be determined whether the elevated sFlt 1 levels in the placenta would not further aggravate the disease by impairing VEGF-dependent pseudo-vasculogenesis and maternal spiral artery remodeling.

What medical implications do these studies suggest? First, strategies designed to normalize circulating free VEGF and PlGF levels might be expected to halt progression of the disease. Second, chronic administration of VEGF inhibitors, currently being evaluated for their therapeutic potential to inhibit cancer, retinal neovascularization, and chronic inflammation, may pose a risk of endothelial dysfunction, renal insufficiency, and hypertension. Results from ongoing clinical trials support this notion. Third, renal parameters and blood pressure might be used as surrogate markers of the success of anti-angiogenesis therapy - the angiogenesis field desperately needs such markers. At the very least, these studies raise new hopes that novel strategies may be designed to combat preeclampsia and thereby improve the chances of the mother and child for a healthy future.

\footnotetext{
1. Roberts, J.M., and Cooper, D.W. 2001. Pathogenesis and genetics of pre-eclampsia. Lancet. 357:53-56.
}

2. Pridjian, G., and Puschett, J.B. 2002. Preeclampsia. 
Part 1: clinical and pathophysiologic considerations. Obstet. Gynecol. Surv. 57:598-618.

3. Podjarny, E., Baylis, C., and Losonczy, G. 1999. Animal models of preeclampsia. Semin. Perinatol. 23:2-13.

4. Maynard, S.E., et al. 2003. Excess placental soluble fms-like tyrosine kinase 1 (sFlt1) may contribute to endothelial dysfunction, hypertension, and proteinuria in preeclampsia. J. Clin. Invest. 111:649-658. doi:10.1172/JCI200317189.
5. Eremina, V., et al. 2003. Glomerular-specific alter ations of VEGF-A expression lead to distinct congenital and acquired renal diseases. J. Clin. Invest. 111:707-716. doi:10.1172/JCI200317423.

6. King, B.F. 1987. Ultrastructural differentiation of stromal and vascular components in early macaque placental villi. Am. J. Anat. 178:30-44

7. Goldman-Wohl, D., and Yagel, S. 2002. Regulation of trophoblast invasion: from normal implantation to pre-eclampsia. Mol. Cell. Endocrinol. 187:233-238.
8. Zhou, Y., et al. 2002. Vascular endothelial growth factor ligands and receptors that regulate human cytotrophoblast survival are dysregulated in severe preeclampsia and hemolysis, elevated liver enzymes, and low platelets syndrome. Am. J. Pathol. 160:1405-1423.

9. Mattot, V., et al. 2002. Loss of the VEGF(164) and VEGF(188) isoforms impairs postnatal glomerular angiogenesis and renal arteriogenesis in mice. J. Am. Soc. Nephrol. 13:1548-1560.

\title{
Nephrolithiasis: site of the initial solid phase
}

\author{
David A. Bushinsky
}

University of Rochester School of Medicine and Dentistry and the Nephrology Unit, Strong Memorial Hospital, Rochester, New York, USA

J. Clin. Invest. 111:602-605 (2003). doi:10.1172/JCI200318016.

Most cases of nephrolithiasis are associated with the relatively common metabolic abnormality of idiopathic hypercalciuria (1). These patients generally absorb an excess amount of dietary calcium leading to increased urine calcium excretion and supersaturation with respect to calcium oxalate and calcium phosphate; they subsequently form stones. Other patients with nephrolithiasis, who have had an intestinal bypass procedure, absorb oxalate in excess leading to increased urine oxalate excretion and supersaturation with respect to calcium oxalate; they also subsequently form stones. In these and other causes of nephrolithiasis, the site of the initial solid phase has long been the subject of debate. Over 65 years ago, A. Randall demonstrated that interstitial crystals located at, or adjacent to, the papillary tip, Randall's plaques, were common in

\footnotetext{
Address correspondence to: David A. Bushinsky, University of Rochester School of Medicine and Dentistry, Nephrology Unit, Strong Memorial Hospital, 601 Elmwood Avenue, Box 675, Rochester, New York 14642, USA. Phone: (585) 275-3660;

Fax: (585) 442-9201; E-mail:

David_Bushinsky@urmc.rochester.edu.

Conflict of interest: The author has declared that no conflict of interest exists.
}

stone formers (2). He found that these crystals were composed not of calcium oxalate, the most common solid phase found in patients with nephrolithiasis, but of calcium phosphate (3). He believed that the calcium phosphate crystals formed in the papillary interstitium and then eroded into the urinary space, serving as a heterogeneous nucleation surface for calcium oxalate. B. Finlayson later argued that, due to rapid flow of the renal ultrafiltrate through the tubule, there was insufficient time for formation of a lumenobstructing solid phase (4), which also suggested that an intratubular site of stone formation was unlikely. However, other investigators found that calcium oxalate crystals adhered to cultured tubular cells (5), where they could either be endocytosed or remain on the cell surface, serving as a nidus for growth into larger, clinically significant, calculi.

\section{Site of the initial solid phase}

Where is the site of initial crystallization - the interstitium, the tubular lumen, or perhaps the renal calyx, where supersaturated fluid awaits excretion into the ureter? Knowing the site of initial crystallization would improve understanding of the patho- genesis of stone formation and allow investigators to propose and test more focused hypotheses. This would help them to devise effective therapy aimed at preventing recurrent nephrolithiasis, which afflicts approximately 50\% of stone formers within five years of the initial stone (6). Yet until the elegant study by A.P. Evan et al. reported in this issue of the JCI (7), we did not have an answer to this rather elementary question. These investigators performed kidney biopsies on stoneforming patients to determine the anatomical site and composition of the initial solid phase. They sampled areas adjacent to Randall's plaques in patients undergoing percutaneous nephrolithotomy. In hypercalciuric calcium oxalate stone formers, they found initial calcium phosphate (apatite) crystallization in the basement membrane of the thin limbs of the loop of Henle (Figure 1) with subsequent extension to the vasa recta, then to the interstitial tissue surrounding the terminal collecting ducts, and finally, in the most severe cases, to the papillae. Erosion of this solid phase into the urinary space, which is supersaturated with respect to calcium oxalate, may have promoted heterogeneous nucleation and formation of kidney stones. In patients with hyperoxaluria resulting from intestinal bypass, the initial crystals were again a calcium phosphate complex, but these arose within the tubule lumens of terminal collecting ducts (Figure 2). Contact of these crystals with urine, supersaturated with respect to calcium oxalate, may have promoted heterogeneous nucleation and formation of kidney stones. Nonstone formers, subjected to nephrectomy, had neither plaque nor crystals. Thus there are different sites of initial 